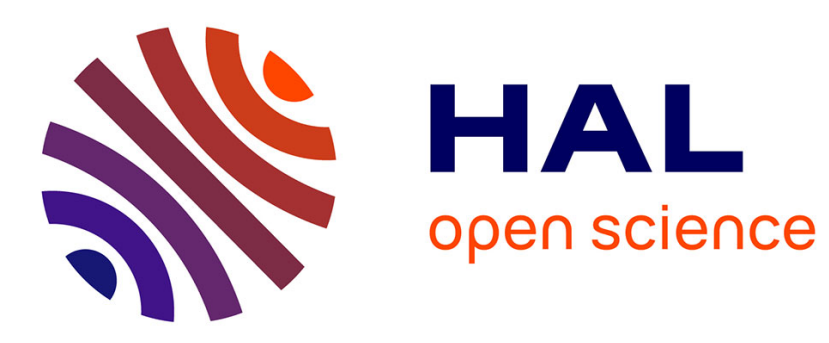

\title{
Providing Collision-Free and Conflict-Free Communication in General Synchronous Broadcast/Receive Networks
}

Abdelmadjid Bouabdallah, Hicham Lakhlef, Michel Raynal, François Taïani

\section{- To cite this version:}

Abdelmadjid Bouabdallah, Hicham Lakhlef, Michel Raynal, François Taïani. Providing Collision-Free and Conflict-Free Communication in General Synchronous Broadcast/Receive Networks. 31st IEEE International Conference on Advanced Information Networking and Applications (AINA 2017), Mar 2017, Taipei, Taiwan. pp.399-406, 10.1109/AINA.2017.39 . hal-01620356

\section{HAL Id: hal-01620356 \\ https://hal.inria.fr/hal-01620356}

Submitted on 20 Oct 2017

HAL is a multi-disciplinary open access archive for the deposit and dissemination of scientific research documents, whether they are published or not. The documents may come from teaching and research institutions in France or abroad, or from public or private research centers.
L'archive ouverte pluridisciplinaire HAL, est destinée au dépôt et à la diffusion de documents scientifiques de niveau recherche, publiés ou non, émanant des établissements d'enseignement et de recherche français ou étrangers, des laboratoires publics ou privés. 


\title{
Providing Collision-free and Conflict-free Communication in General Synchronous Broadcast/Receive Networks
}

\author{
Abdelmadjid Bouabdallah ${ }^{\dagger}$ Hicham Lakhlef ${ }^{\dagger} \quad$ Michel Raynal $^{\ddagger, \star} \quad$ François Taïani $^{\ddagger}$ \\ † Sorbonne universits, Universit de technologie de Compigne, CNRS, \\ Heudiasyc UMR 7253, CS 60319 ; 60203 Compigne Cedex \\ $\ddagger$ IRISA, Université de Rennes, France \\ * Institut Universitaire de France \\ bouabdal@utc.fr hlakhlefeutc.fr raynal@irisa.fr francois.taiani@irisa.fr
}

\begin{abstract}
This work considers the problem of communication in dense and large scale wireless networks composed of resourcelimited nodes. In this kind of networks, a massive amount of data is becoming increasingly available, and consequently implementing protocols achieving error-free communication channels constitutes an important challenge. Indeed, in this kind of networks, the prevention of message conflicts and message collisions is a crucial issue. In terms of graph theory, solving this issue amounts to solve the distance-2 coloring problem in an arbitrary graph. The paper presents a distributed algorithm providing the processes with such a coloring. This algorithm is itself collision-free and conflict-free. It is particularly suited to wireless networks composed of nodes with communication or local memory constraints.
\end{abstract}

Keywords: Arbitrary networks, Broadcast/receive, Collision, Conflict, Distance-2 graph coloring, Message-passing, Network traversal, Synchronous system, Time slot assignment, Wireless network.

\section{INTRODUCTION}

a) Synchronous broadcast/receive systems: In a synchronous system, the processes execute a sequence of rounds, where a round is a bounded time slot such that any message send at the beginning of a slot is received by the end of the same slot by its receiver [15]. In such a system, a collision occurs when a process receives several messages during the same round (time slot), or when two neighbor processes send message to each other during the same round.

A broadcast/receive communication system provides processes with two operations: a broadcast which allows it to send a message to all its neighbors, and a receive which allows it to receive message. This paper considers synchronous system with broadcast/receive communication.

b) Distance-2 coloring: One way to prevent collisions and conflicts in synchronous broadcast/receive systems consists in assigning a color to each neighbor in such a way that no two processes at distance $\leq 2$ have different colors. Let $K$ the maximal number of colors that are used. Let color $_{i}$ be the color of $p_{i}$. Once the distance- 2 coloring is done, a process is allowed to broadcast a message to its neighbors only at the rounds $r$ such that color $_{i}=(r \bmod K)$. It is easy to see that no neighbors processes can broadcast during the same round, and no two neighbors of a process $p_{i}$ can broadcast during the same round.

c) Content of the paper: Several distance-2 coloring distributed algorithms have been proposed in the literature, in a variety of different contexts. Some address synchronous send/receive message-passing systems (e.g., [1], [16]); some address shared memory systems [3], [7]; some address selfstabilizing systems (e.g., [2], [6], [8]; some targeting wireless sensor networks (e.g., [4], [5], [9], [11], [12], [13], [14]). None of them considers the pure synchronous broadcast/receive system model, which we recently addressed in [10], where is presented a distributed distance- 2 coloring algorithm suited to tree networks. This algorithm is optimal in the number of colors, namely it uses $K=\Delta+1$ colors (where $\Delta$ is the maximal degree of the communication graph -maximal number of neighbors of any vertex-).

d) Remark: This paper extends to an arbitrary graph, the algorithm presented in [10] which considers tree networks. Due to the fact that a tree has no cycles, this "extension" is far from being trivial. Nevertheless the model and the problem are the same in both cases. Hence, to have a self-contained paper, parts of Section II and Section III of the present paper are a direct copy-and-paste of the corresponding parts of [10].

This paper extends our work to an connected graph. It is made up of 6 sections. Section II presents the computation model. Section III presents the distance-2 coloring problem. Section IV presents a distributed algorithm realizing a distance- 2 coloring of an arbitrary graph. This algorithm is based on a sequential network traversal [16] enriched with "backtracking" messages used to solve coloring conflicts. Section V presents an execution of the algorithm on a simple graph including a cycle. Finally, Section VI proves its correctness. 


\section{Synchronous BRoAdCASt/Receive Model}

a) Processes, initial knowledge, and the communication graph: The system model consists of $n$ sequential processes denoted $p_{1}, \ldots, p_{n}$, connected by an arbitrary communication graph.

Each process $p_{i}$ has an identity $i d_{i}$, which is known only by itself and its neighbors (processes at distance 1 from it). The constant neighbors $s_{i}$ is a local set, known only by $p_{i}$, including the identities of its neighbors (and only them). As noticed in the Introduction, in order for a process $p_{i}$ not to confuse its neighbors, it is assumed that no two processes at distance less than or equal to 2 have distinct identities. Hence, any two processes at distance greater than 2 may have the same identity. When computing bit complexities, we will assume that any process identity is encoded in $\log _{2} n$ bits.

Let $\Delta_{i}$ denote the degree of a process $p_{i}$ (i.e. $\mid$ neighbors $_{i} \mid$ ) and let $\Delta$ denote the maximal degree of the process graph $\left(\max \left\{\Delta_{1}, \cdots, \Delta_{n}\right\}\right)$. While each process $p_{i}$ knows $\Delta_{i}$, no process knows $\Delta$ (a process $p_{x}$ such that $\Delta_{x}=\Delta$ does not know that $\Delta_{x}$ is $\Delta$ ).

When considering a process $p_{i}, 1 \leq i \leq n$, the integer $i$ is called its index. Indexes are not known by the processes. They are only a notation convenience used as a subscript to distinguish processes and their local variables.

b) Timing model: We assume that processing durations are equal to 0 . This is justified by the following observations: (a) the duration of the local computations of a process is negligible with respect to message transfer delays, and (b) the processing duration of a message may be considered as a part of its transfer delay.

Communication is synchronous in the sense that there is an upper bound $D$ on message transfer delays, and this bound is known by all the processes (global knowledge). From an algorithm design point of view, we consider that there is a global clock, denoted $C L O C K$, which is increased by 1 , after each period of $D$ physical time units. Each value of $C L O C K$ defines what is usually called a time slot or a round.

c) Communication operations: The processes are provided with two operations denoted broadcast () and receive( $)$. A process $p_{i}$ invokes broadcast $\mathrm{TAG}(m)$ to send the message $m$, whose type is TAG, to all its neighbors. It is assumed that a process invokes broadcast() only at a beginning of a time slot. When a message $\operatorname{TAG}(m)$ arrives at a process $p_{i}$, this process is immediately warned of it, which triggers the execution of operation receive() to obtain the message. Hence, a message is always received and processed during the time slot -roundin which it was broadcast.

From a linguistic point of of view, we use the two following when notations when writing algorithms, where predicate is a predicate involving $C L O C K$ and possibly local variables of the concerned process.

when $\operatorname{TAG}(m)$ is received do processing of the message. when predicate do code entailing one broadcast() invocation.

d) Message collision and message conflict: Traditional wired round-based synchronous systems assume a dedicated communication medium for each pair of processes (i.e., this medium is not accessible to the other processes). Hence, in these systems a process $p_{i}$ obeys the following sequential pattern during each round: (a) first $p_{i}$ sends a message to all or a subset of its neighbors, (b) then $p_{i}$ receives the messages sent to it by its neighbors during the current round, and (c) finally executes a local computation which depends on its local state at the beginning of the round and the messages it has received during the current round.

The situation is different in systems such as wireless networks (e.g., sensor networks), which lack a dedicated communication medium per pair of processes. A process $p_{i}$ shares a single communication medium with all its neighbors, and "message clash" problems can occur, each message corrupting the other ones, and being corrupted by them. Consider a process $p_{i}$, these problems are the following.

- If two neighbors of $p_{i}$ invoke the operation broadcast() during the same time slot (round), a message collision

- Pf $p_{i}$ and one of its neighbors invoke broadcast() during the same time slot (round), a message conflict occurs.

As already indicated, this paper considers this broadcast/receive communication model. This implies that protocols must prevent collisions and conflicts to ensure both message consistency and computation progress.

\section{The Distance-2 Coloring Problem}

a) Solving the collision/conflict problem: To prevent collisions and conflicts involving a process $p_{i}$, only a single process in the set $\left\{p_{i}\right\} \cup$ neighbors $_{i}$ can obtain the right to communicate during a given round. To this end, we associate each process with time slots (rounds) in which it can broadcast a message, while none of its 2-hop neighbors can broadcast during these time slots. When considering the whole set of processes, this assignment must be optimal in terms of numbers of colors (ideally allowing as many processes as possible to broadcast during the same round).

This problem is a well-known graph coloring problem called distance-2 coloring. The aim is to design distributed algorithms associating a color with each process (which will define the time-slots during which it will be allowed to broadcast) such that the following properties are satisfied.

b) Definition:

- Validity: The final color of each process belongs to $\{0, \ldots, K\}$, where $K \leq \Delta^{2}$.

- Consistency: No two processes at distance $\leq 2$ have the same color at the end of the coloring algorithm.

- Termination: Each process obtains a color and one process knows that this occurred.

c) Using the colors to define the time slots: The colors obtained by the processes are used as follows, where color $i$ is the color obtained by process $p_{i}$. The time slots (rounds) during which $p_{i}$ is allowed to broadcast a message to its neighbors correspond to the values of $C L O C K$ such that $\left((C L O C K \bmod (K+1))=\right.$ color $\left._{i}\right)$. As we will see, these time slots are different from the time slots used during 
the (sequential and parallel) distributed distance-2 algorithms which are presented below. It follows that these algorithms must provide each process with the (initially unknown) value of $K$.

\section{Sequential Distance-2 COloring of A GRAPH}

This section presents a distributed distance- 2 coloring distributed algorithm for any connected graph. This solution is shown in Algorithm 1. During the execution of this algorithm there are neither message collisions, nor message conflicts. This algorithm is sequential in the sense that its skeleton is a depth-first tree traversal in which the control flow (implemented by appropriate messages) moves sequentially from a process to another one.

Algorithm 1 assumes that a single process receives a message, $\operatorname{START}()$, which defines it as the root of the network. This external message causes the receiving process, $p_{r}$, to simulate the reception of a fictitious message, $\operatorname{COLOR}\left(i d_{i}, i d_{i},-1,0, \emptyset\right)$. This message initiates a depth-first traversal of the network.

a) Messages: The algorithm uses five types of messages: COLOR(), CORRECT(), CORRECTED_COLOR(), RESUME_COLORING () and TERM () . These messages do not have the same number of parameters. As each message is received by all the neighbors of thge sender, its first parameter is the identity of its destination neighbor process. However, even if the message is not destined to it, the process can do local actions (line 10).

These messages implement a depth-first traversal of the network [16]. Each carries the identity of its destination (dest), the identity of its sender (sender). In addition, the message COLOR () carries the color of its sender (sender_cl), the proposed color (proposed_cl) and the color set of the already colored neighbors of the sender (d1colors). The message CORRECT () carries the color of the sender and the set $d 1$ colors. The message CORRECTED_COLOR () carries a second identity of destination dest 2 and the corrected color (corrected_cl). The message RESUME_COLORING () carries destination identity dest and the sender identity sender.

Let us call the messages CORRECT(), CORRECTED_COLOR() and RESUME_COLORING () as backtracking messages.

b) Local variables: Each process $p_{i}$ manages the following local variables.

- state $_{i}$ (initialized to 0 ) is used by $p_{i}$ to manage the progress of the network traversal. Each process may traverse seven different states during the execution of the algorithm. States $1,2,3,4,5$, and 6 are active: a process in state 1 broadcasts a $\operatorname{COLOR}()$ message destined to one of its uncolored children, a process in state 2 broadcasts a CORRECT() message destined to its parent after the detection of a temporary inconsistency in the coloring, a process in state 3 broadcasts a TERM () message destined to its parent, a process in state 4 or 6 broadcasts a CORRECTED_COLOR () message, and a process in state 5 broadcasts a RESUME_COLORING() message to its child.
States 0 is a waiting state. Nodes listen on the broadcast channel but cannot send any message.

- parent $_{i}$ saves the identity of the process $p_{j}$ from which $p_{i}$ received the message $\operatorname{COLOR}\left(i d_{i},-,-,-,-\right) ; p_{i}$ receives exactly one such message. This process, $p_{j}$, defines the parent of $p_{i}$ in the built tree. The root $p_{r}$ of the built tree, defined by the reception of the external message $\operatorname{START}()$, is the only process such that parent $_{r}=i d_{r}$.

- sender $_{-} l_{i}$ records the color of the parent of $p_{i} . p_{i}$ receives this information in the parent's COLOR message.

- d1 $_{\text {colors }}$ is a set containing the colors of the neighbors of $p_{i}$, that have already obtained their color.

- d2 colors $_{i}$ is a set containing the colors of the neighbors of neighbors of $p_{i}$ (processes at distance 2), that have already obtained their color.

- to_color $i$ (initialized to neighbors ${ }_{i}$ ) is a set containing the identities of the neighbors of $p_{i}$ not yet colored.

- color $_{i}$ contains the color of $p_{i}$.

c) Description of the algorithm: Processes start the algorithm in state 0 , waiting for a $\operatorname{COLOR}\left(i d_{i},-,-,-, \emptyset\right)$ message, where the sender (color proposer) proposes a color to one of its neighbors. A process, $p_{i}$, receives such a message destined to it (i.e dest $=i d_{i}$ ) exactly once. When it receives this message, it is visited for the first time by the depth-first tree traversal. It consequently assigns the values of the message parameters to its local variables parent $_{i}$, sender_cl $l_{i}, d 1$ colors $_{i}$ and d2 $_{\text {colors }}$ (lines 02, 06, 07). If the proposed color is not used by the neighbors and the neighbors of neighbors, and the color of the color proposer is not used by any process in neighbor $s_{i}$, then the process accepts the proposed color (line 06) and moves to the state 1 . So it can broadcast a COLOR() message at line 13 if it has at least one uncolored neighbor. This message is destined to this uncolored neighbor (line 12). At line 07 , if a process, $p_{i}$, does not have neighbors to color, it obtains state $_{i}=3$. This state allows the process to broadcast in the next round a TERM () message destined to its parent. And if all the children of a parent are colored (line 24), this parent obtains state $_{i}=3$ which permits to it the broadcast of $\operatorname{TERM}()$ message destined to its parent and so on following lines 18, 25. Otherwise (there is an uncolored neighbor), the parent obtains the state 2 which allows, in the next round, the broadcast of a $\operatorname{COLOR}()$ message destined to one of its uncolored neighbors.

If a destination process, $p_{i}$, detects that the color of the color proposer is used by one of its neighbors, or the proposed color is used by a neighbor or a process at distance 2 , then it does not take the proposed color, and moves to state $_{i}=2$ ( lines 03 and 04). This state allows it to inform the color proposer about this temporary inconsistency using a CORRECT() message at line 11. At the reception of this message, the process updates

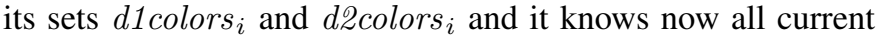
colors of the processes at distance 2 (lines 29). At line 30 it then obtains a new color which is not used by its neighbors and the processes at distance 2 . It also obtains state $_{i}=4$ which permits it to broadcast a CORRECTED_COLOR() message to its neighbors (line 19). At the reception of this message the 


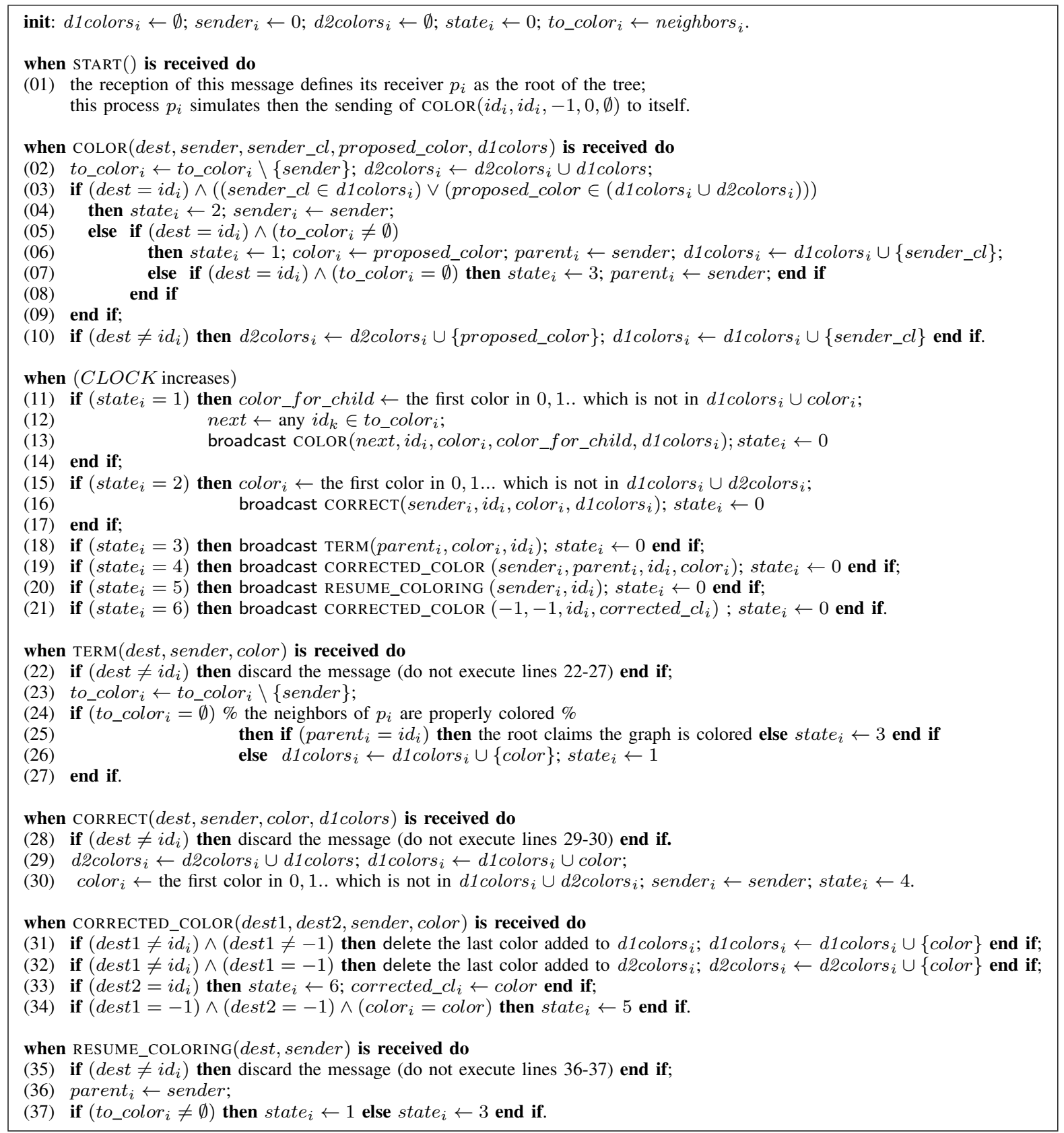

Algorithm 1: Sequential distance-2 coloring for an arbitrary graph (code for $p_{i}$ )

neighbors delete the previous colors added in the previous round to the lists $d 1$ colors $_{i}$ and $d 2$ colors $_{i}$, and replace them with the corrected colors (lines 31 and 32).

When the message CORRECTED_COLOR () is received by the parent of the process that detected the temporary inconsistency it obtains the state 3 in line 34. This state allows to this process the broadcast of RESUME_COLORING () message destined to its child that sent the message CORRECT() (line 20). At the reception of this message, the process obtains the state 2 if it has neighbors to color, else it obtains the state 3 (signaling local termination) which allows the broadcast of a $\operatorname{TERM}()$ message destined to its parent.

\section{A Simple ExAmple}

Considering the network of Figure 1, an example of execution of Algorithm 1 is depicted in Table I. Due to space restriction we abbreviate the following:

- br $=$ broadcast operation,

- $d 1_{i}=d 1$ colors $_{i}$,

- $d 2_{i}=d 2$ colors $_{i}$,

- $\operatorname{CL}\left(p_{a}, p_{b}, \mathrm{c}, \mathrm{z}, \mathbf{S}\right)=\operatorname{COLOR}\left(p_{a}, p_{b}, \mathrm{c}, \mathbf{z}, \mathbf{S}\right)$,

- $\operatorname{CRL}\left(p_{a}, p_{b}, \mathbf{c}, \mathbf{S}\right)=\operatorname{CORRECT}\left(p_{a}, p_{b}, \mathbf{c}, \mathbf{z}, \mathbf{S}\right)$,

- CR_CL $\left(p_{a}, p_{b}, \mathrm{c}, \mathbf{S}\right)=$ CORRECTED_CL $\left(p_{a}, p_{b}, \mathrm{c}, \mathbf{S}\right)$,

- RSM_CL $\left(p_{a}, p_{b}\right)=\operatorname{RESUME} \_\mathrm{CL}\left(p_{a}, p_{b}\right)$, 


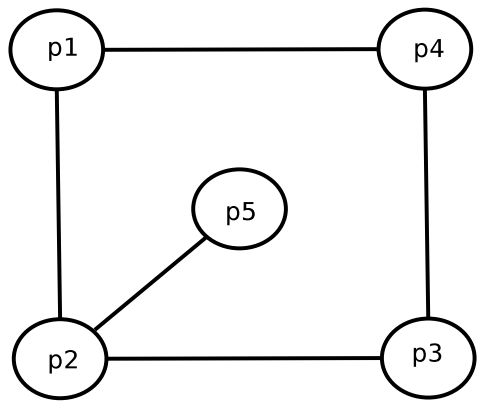

Figure 1. A 5-process arbitrary network

- $s_{i}=$ state $_{i}$.

Process $p_{1}$ receives the message $\operatorname{START}()$ at round -1 . It broadcasts the message COLOR $\left(i d_{1}, i d_{1},-1,0, \emptyset\right)$ to itself. It receives this message at round 0 . It updates its local variables $d 2_{1}$ at line 02 (abbreviated as L2), $d 1_{1}, s_{1}$ and color ${ }_{1}$ at line 06 (abbreviated as L6). This appears in the first row of the table where the value of $C L O C K$ is 0 . Then, when $C L O C K$ progresses to $1, p_{1}$ has state $_{1}=1$, therefore, it broadcasts the message $\operatorname{COLOR}()$ with appropriate parameters, where it proposes a color that is not in $d 2_{1} \cup d 1_{1}$ to each of its neighbors, and subsequently enters state $_{1}=0$ (L13). This appears in the second row of the table where the value of $C L O C K$ is 1 . When $p_{2}$ and $p_{4}$ receive this message at round 2 , they execute the associated processing at L2 and L6 for $p_{2}$ and at L10 for $p_{4}$. So, in this round $p_{2}$ updates its local variables $d 2_{2}$ at $\mathrm{L} 2, d 1_{2}, s_{2}$ and color $_{2}$ at L6. $p_{4}$ updates its local variables $d 2_{4}$ at $\mathrm{L} 2, d 1_{4}$, at L10. This appears in the third row of the table where $C L O C K$ is 2 . In round $3, p_{2}$ has state $_{2}=1$, so it broadcasts the message $\operatorname{COLOR}()$ with appropriate parameters (L13) and obtains state $_{2}=0$ (L13), Etc.

In round $6, p_{4}$ finds that the color proposed by its neighbor $p_{3}$ (color 0 proposed in round 5 ) is in $d 1_{4}$, so it "refuses" this color and obtains state $_{4}=2(\mathrm{~L} 4)$. In round 7, $p_{4}$ obtains a color which is not in $d 2_{4} \cup d 1_{4}$ (L15) and broadcasts the message CORRECT $\left(i d_{3}, i d_{4}, 2,0,\{0\}\right)$. In round $8, p_{3}$ updates $d 1_{3}$ and $d 2_{3}$ and obtains a new color (L30). Its state will allow it to broadcast the message CORRECTED_CL() (round 11). The broadcast of this message will trigger the broadcast of the message RESUME_CL() (round 15) to resume the coloring as described before.

\section{Proof And Cost of the Algorithm}

Lemma 1. Algorithm 1 is collision-free and conflict free.

Proof Let us recall that, in state 0 , a process cannot broadcast messages. The states in $S=\{1,2,3,4,5,6\}$ are active states. Let us observe that after the reception of the message START(), there is only one process (the initiator) that can broadcast a message to its neighbors in the next round (it enters state 1, line 06). The other processes are initially in state 0 , which prevents them from broadcasting a message.

Let us now observe that, after the reception of a message $m$, a process $p_{i}$ can change its state in two cases:
- Case 1 . The message $m$ is for it $\left(d e s t=i d_{i}\right)$, or

- Case 2. $m=$ CORRECTED_COLOR $(-1,-1,-$, color $)$, where color $_{i}=$ color .

In the first case the sender process puts only one identity destination in each message, due to lines 04,06 and 12. Let us observe that $p_{i}$ cannot move to an active state if it is not the destination of message sent from one of its neighbors, $p_{j}$ (lines 03, 05, 07, 22, 28, 31, 35). The broadcasting of this message by $p_{j}$ entails the immediate assignment state $_{j} \leftarrow 0$. It follows that the sender process and the destination process cannot broadcast in the same round. Each time a message is broadcast by a process $p_{j}$, there is only one neighbor of $p_{j}$ that will move to an active state. Therefore, at each round there is no two processes $p_{i}$ and $p_{q}$ in neighbor $s_{j}$ that have state $_{i}$ and state $_{q}$ in $S$. It follows that there will be only one neighbor of $p_{j}$ authorized to broadcast a message in the next round.

In the second case we have to prove that there is only one process that moves to the state 5 after the broadcast of the message CORRECTED_COLOR $(-1,-1,-$, color $)$. Let $p_{f}$ be the the sender of this message. Since $p_{f}$ is broadcasting this message, it has the state 6. It got this state in the previous round because it has received message CORRECTED_COLOR $\left(-, i d_{f},-,-\right)$ from its child $p_{z}$ that has color $_{z}=$ color . Let us assume that there is another process $p_{x} \in$ neighbor $_{f}$ that has color $x=$ color. If $p_{x}$ is the process that broadcast a $\operatorname{COLOR}()$ message destined to $p_{f}\left(p_{x}\right.$ colored $p_{f}$ ), then due to line $11 p_{f}$ does not propose the color color to its children. And if $p_{f}$ is the process that broadcast a COLOR () message to $p_{x}$, then $p_{z}$ is aware about that (line 10) and know that the color color is used by a process at distance 2. Therefore, $p_{z}$ cannot accept this color and moves to the state 2 to correct the proposition. It follows that the message CORRECTED_COLOR $(-1,-1,-$, color $)$ is destined to only one neighbor.

Hence, the control flow generated by these messages remains sequential, moving sequentially from a parent process to a child process for the messages $\operatorname{COLOR}()$, CORRECTED_COLOR( () and RESUME_COLORING(). Similarly, the control flow generated by the messages $\operatorname{TERM}()$ and CORRECT () moves sequentially from a child process $p_{i}$ to its parent process (whose identity is saved in parent ${ }_{i}$ ).

The collision-freedom and conflict-freedom properties of the algorithm follow directly from the sequentiality of the control flow realized by the messages.

$\square$ Lemma 1

Lemma 2. No two processes at distance $\leq 2$ obtain the same color.

Proof Let us observe that the initiator takes the first color 0 (line 01) and each process may propose in a $\operatorname{COLOR}()$ message one color to only one of its uncolored neighbors (lines 11-14).

Let us recall that each process in the network receives messages only from its one hop neighbors. Therefore, only neighbors know if a given color is proposed to neighbors of neighbors (line 10). Due to the instruction in line 11, a process 


\begin{tabular}{|c|c|c|c|c|c|}
\hline $\begin{array}{l}p_{i} \\
\text { clock }\end{array}$ & $p_{1}$ & $p_{2}$ & $p_{3}$ & $p_{4}$ & $p_{5}$ \\
\hline 0 & $\begin{array}{l}d 2_{1}=\{\}(\mathrm{L} 2), d 1_{1}=\{-1\} \\
s_{1}=1, \text { color }_{1}=0(\mathrm{~L} 6)\end{array}$ & & & & \\
\hline 1 & $\begin{array}{l}\text { br CL }\left(i d_{2}, i d_{1}, 0,1,\{-1\}\right) \\
s_{1}=0(\mathrm{~L} 13)\end{array}$ & & & & \\
\hline 2 & & $\begin{array}{l}d 2_{2}=\{-1\}(\mathrm{L} 2), d 1_{2}=\{0\} \\
s_{2}=1, \text { color }_{2}=1(\mathrm{~L} 6)\end{array}$ & & $\begin{array}{l}d 2_{4}=\{1\}, d 1_{4}=\{0\} \\
(\mathrm{L} 10)\end{array}$ & \\
\hline 3 & & $\begin{array}{l}\text { br CL }\left(i d_{3}, i d_{2}, 1,2,\{0\}\right) \\
s_{2}=0(\mathrm{~L} 13)\end{array}$ & & & \\
\hline 4 & $\begin{array}{r}d 2_{1}=\{-1,2\} \\
d 1_{1}=\{1\}(\text { L } 10)\end{array}$ & & $\begin{array}{l}d 2=\{0\}(\mathrm{L} 2), d 1_{3}=\{1\} \\
s_{3}=1, \text { color } 3=2(\mathrm{~L} 6)\end{array}$ & & $\begin{array}{c}d 2_{5}=\{0,2\} \\
d 1_{5}=\{1\}(\text { L10) }\end{array}$ \\
\hline 5 & & & $\begin{array}{l}\text { br CL }\left(i d_{4}, i d_{3}, 2,0,\{1\}\right) \\
s_{3}=0(\mathrm{~L} 13)\end{array}$ & & \\
\hline 6 & & $\begin{array}{l}d 2_{2}=\{-1,0\}, \\
\{0,2\}(\text { L } 10)\end{array}$ & & $\begin{array}{l}d 2_{4}=\{1\} \quad(\mathrm{L} 2), \\
s_{4}=2(\mathrm{~L} 4)\end{array}$ & \\
\hline 7 & & & & $\begin{array}{l}\text { color }_{4}=2 \text { (L15), } \\
\text { br CR }\left(i d_{3}, i d_{4}, 2,\{0\}\right) \\
s_{4}=0(\mathrm{~L} 16)\end{array}$ & \\
\hline 8 & & & $\begin{array}{l}d 2_{3}=\{0\} \\
d 1_{3}=\{1,2\}(\mathrm{L} 30) \\
\text { color }_{3}=3, s_{3}=4(\mathrm{~L} 31)\end{array}$ & & \\
\hline 9 & & & $\begin{array}{l}\text { br CR_CL }\left(i d_{4}, i d_{2}, i d_{3}, 3\right) \\
s_{3}=0(\mathrm{~L} 19)\end{array}$ & & \\
\hline 10 & & $\begin{array}{l}d 1_{2}=\{0,3\}(\mathrm{L} 31), \quad s_{2}= \\
6(\mathrm{~L} 33)\end{array}$ & & & \\
\hline 11 & & $\begin{array}{l}\text { br CR_CL }\left(-1,-1, p_{2}, 3\right) \\
s_{2}=0 \text { (L21) }\end{array}$ & & & \\
\hline 12 & $d 2_{1}=\{-1,3\}(\mathrm{L} 32)$ & & & & \\
\hline 13 & & & $s_{3}=5(\mathrm{~L} 34)$ & & $d 2_{5}=\{0,3\}(\mathrm{L} 32)$ \\
\hline 14 & & & $\begin{array}{l}\text { br RSM_CL }\left(i d_{4}, i d_{3}\right) \\
s_{3}=0(\text { L20) }\end{array}$ & & \\
\hline 15 & & & & $s_{4}=3(\mathrm{~L} 37)$ & \\
\hline 16 & & & & $\begin{array}{l}\text { br TERM }\left(i d_{3}, i d_{4}\right) \\
s_{4}=0(\mathrm{~L} 18)\end{array}$ & \\
\hline 17 & & & $s_{3}=3(\mathrm{~L} 37)$ & & \\
\hline 18 & & & $\begin{array}{l}\text { br TERM }\left(i d_{2}, i d_{3}\right) \\
s_{3}=0(\mathrm{~L} 18)\end{array}$ & & \\
\hline 19 & & $\begin{array}{l}s_{2}=1(\mathrm{~L} 26) \\
d 1_{2}=\{0,3\}(\mathrm{L} 26)\end{array}$ & & & \\
\hline 20 & & $\begin{array}{l}\operatorname{brCL}\left(i d_{5}, i d_{2}, 1,2,\{0,3\}\right)(\mathrm{L} 16) \\
s_{2}=0(\mathrm{~L} 13)\end{array}$ & & & \\
\hline 21 & $\begin{array}{l}d 2_{1}=\{-1,3,2\} \\
d 1_{1}=\{0,1\}(\text { L10) }\end{array}$ & & $\begin{array}{l}d 2_{3}=\{0,2\} \\
d 1_{3}=\{2,1\}(\mathrm{L} 10)\end{array}$ & & $\begin{array}{l}d 2_{5}=\{0,3\} \quad s_{5}= \\
3(\mathrm{~L} 07)\end{array}$ \\
\hline 22 & & & & & $\begin{array}{l}\operatorname{br} \operatorname{TERM}\left(i d_{2}, i d_{5}\right) \\
s_{5}=0(\mathrm{~L} 18)\end{array}$ \\
\hline 23 & & $s_{2}=3(\mathrm{~L} 25)$ & & & \\
\hline 24 & & $\begin{array}{l}\text { br TERM }\left(i d_{1}, i d_{2}\right) \\
s_{2}=0(\mathrm{~L} 18)\end{array}$ & & & \\
\hline 25 & end algorithm (L25) & & & & \\
\hline
\end{tabular}

Table I

AN EXECUTION OF Algorithm 1 ON THE NETWORK OF Figure 1

proposes to its neighbors colors which are different from its color and different from the colors of its colored neighbors.

Although each process, $p_{j}$, does not propose color $_{j}$ and d1 1 colors $_{j}$, a colored process $p_{i}$ at distance 2 from a colored process $p_{j}$ may propose the same color of $p_{j}$ to one of its uncolored neighbors $p_{e}$ which is at distance 2 from $p_{j}$. Which violates the consistency propriety. Also, a non-colored process $p_{e}$ that has at least two colored neighbor $p_{i}$ and $p_{j}$ may receive as proposed color the color of $p_{j}$ from $p_{i}$ if $p_{i}$ is not $p_{j}$ 's neighbor. Which creates two neighbors with the same color and violates the consistency propriety. This cases may occur because the process does not see the process at distance 1 or 2 of its neighbors.

However, due to line 03 any violation of this kind is detected. Because the color of $p_{j}$ is already stored in $d 1$ colors $_{i}$ and the colors of the neighbors of $p_{j}$ are already stored in d2 colors $_{i}$ (line 10). If the test of the instruction in line 03 is true for $p_{i}, p_{i}$ will not be able to propose a color (broadcast a color message) because its state now is state $_{i}=2$. The possession of this state entails the broadcast of a CORRECT() message to correct the temporary inconsistency by $p_{e}$ that obtains a new color which is different from the colors of its neighbors and the neighbors of its neighbors (line 15).

Due to the instruction in (line 30), the process correct its color based on the information sent by the process that detected the inconsistency and moved to state $_{i}=4$. This state permits to $p_{e}$ to broadcast a CORRECTED_COLOR() message to inform its neighbors about the corrected colors (line 19). Due to the instruction in (line 33), the broadcast of the CORRECTED_COLOR() message entails the the broadcast 
of another CORRECTED_COLOR message by one neighbor (the parent) of $p_{e}$ to inform its neighbor about the new color of its child. And this broadcast entails the broadcast of RESUME_COLORING() message destined to the process $p_{i}$ that detected the inconsistency. Therefore, the process $p_{i}$ that detected the inconsistency cannot take the state $_{i}=1$ ((line 37) until all process which are at distance 2 for the corrected process are have received the message CORRECTED_COLOR(). It follows that before $p_{i}$ resumes the coloring, the current network is colored partially correctly. These complete the proof the lemma.

Lemma 3. Any process is colored, and (only after all processes are colored) this is known by the root process.

Proof If the network is such that there is no temporary inconsistency: in this case due to the instruction in line 06, after the reception of the message color, the process broadcasts in the next round a color message destined to one of its uncolored neighbors. Due to lines 06 and 24, a process does not broadcast a TERM () message if it has at least one uncolored neighbor because it cant not move to the state 3 . It follows that before the root receive all $\operatorname{TERM}()$ messages from all its neighbors each process receives a message color.

If the network is such that there is temporary inconsistency: in this case let us observe that the process $p_{i}$ that detected the temporary inconsistency does not broadcast a $\operatorname{COLOR}()$ message in the next round because it does not have the state 1 (line 04). Following line 16, it broadcasts a CORRECT() message destined to its parent $p_{z}$. Due to line 33 and line $21 p_{z}$ receives the message corrected_color $(-1,-1,-$, color $)$ broadcast by its parent (as discussed in the proof of lemma 1) which allows the broadcast of the message RESUME_COLORING() destined to its child $p_{i}$. Then, at the reception of this message, $p_{i}$ moves to the state 1 . Therefore, $p_{i}$ resume, in a finite time, the coloring at line 11 .

Due to line 25 each process that have all neighbors colored broadcast a $\operatorname{TERM}()$ message destined to its parent.

It follows from these observations that each process eventually obtains one color and the root receives a term message from each neighbors.

$\square_{\text {Lemma } 3}$

The following theorem is an immediate consequence of the previous lemmas.

Theorem 1. Algorithm 1 is a collision-free and conflict-free distance-2 coloring algorithm for any connected graph.

a) Cost of the algorithm: (Let us recall that a process identity can be encoded with $O(\log n)$ bits.) There are five message types. A message TERM () carries two process identities and a color. A message $\operatorname{COLOR}()$ carries two process identities, two colors, and set of at most $\Delta$ colors. A message CORRECT() carries two process identities, a color, and set of at most $\Delta$ colors. A message CORRECTED_COLOR () carries three process identities, and two colors. A message RESUME_COLORING () carries two process identities. It follows that a message carries at most $9 \log n+(2 \Delta+6) \log \Delta$ bits.

Let us observe that each process in the network receive only one message color destined to it.

Let us assume an execution of the algorithm in a network where there will be not a call to broadcast the messages of backtracking (no temporary conflict of colors). Therefore, in this case there are $n-1$ of $\operatorname{COLOR}()$ messages (the initiator does not receive such a message from its neighbors) and $n-1$ of TERM () messages (the last colored process in the network does not receive a TERM () message destined to it). This means there is two messages per each link of the tree built. Therefore, in this best case $2(n-1)$ broadcasts are required.

Let us assume now that the network is such that there is temporary conflicts of colors ( there are calls to the broadcast of backtracking messages.) In this case, there is, in the worst case, four more messages per link: a message CORRECT(), two messages CORRECTED_COLORING() and a message RESUME_COLORING(). Therefore, the number of broadcasts required in the worst case is bound by $6(n-1)$.

\section{CONCLUSION}

There is a major difficulty in designing of a efficient communication protocols when these protocols concern networks connected in an arbitrary manner without any condition of the topology nor on the size of the network, nor on the node characteristics.

An interesting open problem is the following: genalize the previous algorithm solution to a $m$-coloring problem, where a process can get more than one color. And how can we derive, starting from this protocol, solutions that deal with dynamic of the network, in the sense mobility and appearance and disappearance of processes.

\section{ACKNOWLEDGMENTS}

The authors wish to express their appreciation to the anonymous reviewers for their constructive comments.

The authors wish also to express their appreciation to the Labex MS2T for its support.

\section{REFERENCES}

[1] Barenboim L., Elkin M., and Kuhn F., Distributed (Delta+1)-coloring in linear (in Delta) time. SIAM Journal of Computing, 43(1):72-95, 2014.

[2] Blair J. and Manne F., An efficient self-stabilizing distance-2 coloring algorithm. Proc. 16th Colloquium on Structural Information and Communication Complexity (SIROCCO'10), Springer LNCS 5869, pp. 237 251, 2009.

[3] Bozdag D., Gebremedhin A.S., Manne F., Boman G. and Çatalyürek U.V., A framework for scalable greedy coloring on distributed-memory parallel computers. Journal of Parallel and Distributed Computing, 68(4):515-535, 2008.

[4] Chipara O., Lu C., Stankovic J., and Roman. G.-C., Dynamic conflictfree transmission scheduling for sensor network queries. IEEE Transactions on Mobile Computing, 10(5):734-748, 2011.

[5] Ergen S.C. and Varaiya P., PEDAMACS: Power efficient and delay aware medium access protocol for sensor networks. IEEE Transaction on Mobile Computing, 5(7):920-930, 2005.

[6] Gairing M., Goddard W, Hedetniemi S. T., Kristiansen P., and McRae A. A., Distance-two information in self-stabilizing algorithms. Parallel Processing Letters, 14(03-04):387-398, 2004.

[7] Gebremedhin A.H., Manne F., and Pothen A., Parallel distance- $k$ coloring algorithms for numerical optimization. Proc. Euro-Par Parallel Processing, Springer LNCS 2400, pp. 912-921, 2002. 
[8] Herman T., Tixeuil S., A distributed TDMA slot assignment algorithm for wireless sensor networks. Proc. Int'l Workshop on Algorithmic Aspects of Wireless Sensor Networks (ALGOSENSORS'04), Springer LNCS 3121, pp. 45-58, 2004.

[9] Jemili I., Ghrab D., Belghith A., Derbel B., and Dhraief A., Collision aware coloring algorithm for wireless sensor networks. In Proc. 9th Int' lWireless Communications and Mobile Computing Conference (IWCMC'13), pages 1546-1553, 2013.

[10] Frey D., Lakhlef H., and Raynal M., Optimal collision/conflict-free distance-2 coloring in wireless broadcast/receive synchronous tree networks. Proc. 45th Annual Conference on Parallel Processing (ICPP'16), pp. 350-359, 2016.

[11] Mahfoudh S., Chalhoub G., Minet P., Misson M., and Amdouni I., Node coloring and color conflict detection in wireless sensor networks. Future Internet, 2(4):469, 2010.

[12] Prabhakar B., Uysal-Biyikoglu, E., and El Gamal A., Energy-efficient packet transmission over a wireless link. IEEE/ACM Transactions on Networking, 10(4):487499, 2002

[13] Ramanathan S., A unified framework and algorithms for (T/F/C)DMA channel assignment in wireless networks. Proc. 16th IEEE INFOCOM Conference, IEEE Press, pp. 900-907, 1997.

[14] Lakhlef H., Bourgeois J., Harous S., Myoupo J.: Collision-Free Routing Protocol in Multi-hop Wireless Sensor Networks. In CIT 2015, The 15th IEEE Int. Conf. on Computer and Information Technology, Liverpool, UK, pages 9299, October 2015

[15] Raynal M., Fault-tolerant agreement in synchronous message-passing systems. Morgan \& Claypool Publishers, 165 pages, 2010 (ISBN 9781-60845-525-6).

[16] Raynal M., Distributed algorithms for message-passing systems. Springer, 500 pages, 2013, ISBN 978-3-642-38122-5. 